\title{
High speed unsupervised fluorescence lifetime imaging confocal multiwell plate reader for high content analysis
}

C. B. Talbot ${ }^{*}$, , J. McGinty ${ }^{1}$, D. M. Grant ${ }^{2}$, E. J. McGhee ${ }^{1}$, D. M. Owen ${ }^{2}$, W. Zhang ${ }^{3}$, T. D.

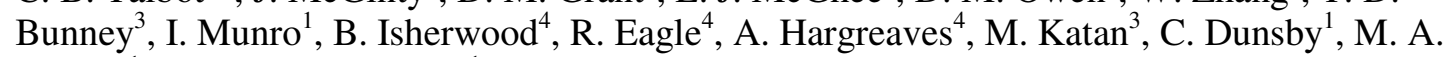
A. Neil ${ }^{1}$ and P. M. W. French ${ }^{1}$

${ }^{1}$ Photonics Group, Imperial College London, London SW7 2AZ, UK

${ }^{2}$ Chemical Biology Centre, Imperial College London, London SW7 2AZ, UK

${ }^{3}$ The Institute of Cancer Research, London SW3 6JB, UK

${ }^{4}$ Advanced Science and Technology Laboratory, AstraZeneca, Loughborough, LE11 5RH

Corresponding author e-mail: clifford.talbot@imperial.ac.uk

Key words: Fluorescence lifetime imaging, time-resolved imaging, high content analysis, unsupervised acquisition.

\begin{abstract}
Abstrcat:
We report an automated optically sectioning fluorescence lifetime imaging (FLIM) multiwell plate reader for high content analysis (HCA) in drug discovery and accelerated research in cell biology. The system utilizes a Nipkow disc confocal microscope and performs unsupervised FLIM with autofocus, automatic setting of acquisition parameters and automated localisation of cells in the field of view. We demonstrate its applications to test dye solutions, fixed and live cells and FLIMFRET.
\end{abstract}

Fluorescence lifetime imaging (FLIM) is a powerful and robust tool, able to contrast different chemical species and sense variations in fluorophore micro-environments [1]. In general, it is a robust quantitative imaging modality that is insensitive to artefacts arising from, e.g. fluorophore concentration, scattering, inner filter effects, etc, that can compromise intensity measurements. FLIM is widely applied to cell biology, particularly to study protein-protein interactions using Forster Resonance Energy Transfer (FRET) [2], as well as to read-out fluorescence-based sensors e.g. di4-ANEPPDHQ to probe lipid order in the cellular membrane [3], and to provide labelfree contrast in biological tissue, e.g. [4]. These capabilities have many potential applications for high content analysis (HCA) but, until recently, a major obstacle to the application of FLIM in this context has been the speed of data acquisition. For cell biology experiments, the most commonly used commercial FLIM systems are based on time correlated single photon counting (TCSPC) implemented in a laser scanning confocal or multiphoton microscope. While this approach has been combined with a multiwell plate reader [5], this instrument did not acquire images but delivered a single lifetime measurement per well. The imaging speed of TCSPC FLIM is limited by the constraints of single photon counting detection and by the nonlinear photobleaching and photodamage that ensues as the power of the scanning laser beam is increased. Wide-field FLIM achieves much faster imaging rates with lower photobleaching due to the parallel pixel interrogation. An automated instrument for unsupervised FLIM was recently reported exploiting frequency domain fluorescence lifetime measurements, which provided an elegant demonstration of the application to FRET and the new opportunities afforded by statistical analysis of such FLIM array data [6]. This system did not, however, provide optical sectioning, which is important for quantitative imaging and is inherent in laser scanning confocal/multiphoton 
systems. We recently demonstrated that wide-field time-gated imaging using a gated optical intensifier (GOI) can be combined with a Nipkow disc confocal microscope to provide high-speed optically sectioned FLIM that is significantly faster, for a given signal to noise ratio $(\mathrm{S} / \mathrm{N})$, than current TCSPC instrumentation and which we demonstrated to acquire time-gated FLIM-FRET images of live cells labelled with FRET constructs at up to 10 frames per second [7]. Although wide-field time-gated FLIM yields a lower S/N per photon emitted by the sample compared to TCSPC, the parallelism provides a greater $\mathrm{S} / \mathrm{N}$ per unit acquisition time, which is important for studying dynamics and for achieving a higher throughput. Here we report an automated high-speed optically sectioned FLIM multiwell plate reader for HCA, including higher throughput (unsupervised) FLIM-FRET, demonstrating its application to both fixed and live cells.

This multiwell plate reader utilises the high-speed Nipkow FLIM instrumentation described in [7], implemented on an automated microscope (Olympus, IX81) that includes a motorised stage (Marzhauser Wetzlar GmbH, Scan IM) and an optical auto focus module. This is controlled by software written in-house in LabVIEW (National Instruments) that automatically focuses the microscope, finds cells, centres them in the field of view and sets the FLIM acquisition parameters. The system utilises a Nipkow disc head (Yokogowa, model CSU10) to provide optical sectioning. Excitation is provided by a fibre-laser-pumped supercontinuum source (Fianium UK Ltd, SC450-6) that produces $\sim 10$ ps pulses at $50 \mathrm{MHz}$ repetition and $\sim 2 \mathrm{~mW} / \mathrm{nm}$ spectral density. It features electronically controlled wavelength selection that is implemented using a home-built prism and motorised slit assembly. Time-resolved imaging is provided by a gated optical intensifier (Kentech Instruments Ltd, model HRI) that is read out using a cooled CCD camera (Hamamatsu Photonics, model ORCA-ER). The HRI was operated with $1 \mathrm{~ns}$ wide gates triggered at various delay times after the excitation pulses to temporally sample the fluorescence decay profiles for each pixel in the field of view. Following the automated data acquisition, single exponential decay curves were fitted to the data to obtain fluorescence lifetimes and the total fluorescence intensity was calculated by summing the time gated images. We typically represent our lifetime data as a single fluorescence lifetime "image" with colour representing the lifetime and brightness representing the total fluorescence intensity. Where quoted, fluorescence lifetimes refer to the mean \pm standard deviation over the pixels within a region of interest.

A key step for this automated FLIM acquisition is setting the exposure time of the read-out camera. Since the dark current of the camera is very low, $\left(0.1 \mathrm{e}^{-}\right.$per second, compared to the $\sim 18000 \mathrm{e}^{-}$well capacity), the $\mathrm{S} / \mathrm{N}$ depends on the total signal detected, rather than the integration time. To obtain the best $\mathrm{S} / \mathrm{N}$, the exposure time should therefore be set such that the CCD camera is just saturated by the brightest pixels in the earliest time gate. To do this automatically, a calibration image is recorded with a short $(\sim 100 \mathrm{~ms})$ CCD integration time, from which the optimum settings are determined according to a pre-measured calibration curve. This preoptimisation of the acquisition settings means that the same $\mathrm{S} / \mathrm{N}$ is obtained in each well of the sample array, which is advantageous since the photon statistics will be consistent between wells. We note that, if the highest $\mathrm{S} / \mathrm{N}$ is not necessary, we can set the auto-expose to use e.g. half the dynamic range of the CCD camera, thereby reducing the integration time and the total acquisition time. 
Figure 1 shows FLIM data of an array of fluorescein and rhodamine B mixtures dissolved in ethanol with varying ratios in a 96-well plate. To test the autoexposure algorithm, acquisitions were repeated with a neutral density (ND) filter placed in the beam path between the Nipkow microscope head and the sample (i.e. situated in both the excitation and emission path) to vary the fluorescence flux. The $\%$ ratio in figure 1(b) refers to the relative fluorescence emission intensity when excited at $488 \mathrm{~nm}$, rather than the relative fluorophore concentrations. This figure illustrates how the auto-exposure algorithm can automatically adjust the integration time of the camera, such that the images are similar in intensity despite the changes in flux, and illustrates that achieving the same dynamic range for each measurement results in consistent standard deviations of the lifetime distributions for each fluorophore mixture.

Figure 1: A montage of (a) time-gated and (b) FLIM images from nine separate acquisitions of fluorescein and rhodamine B. The fluorescein to rhodamine $B$ ratio changes from left to right as indicated and the fluorescence intensity was decreased by placing ND filters in the beam path. The first time gated images (a) have similar intensity and the lifetime maps $(b)$ show consistent data in each column.

A potential challenge for automated wide-field time-gated FLIM is the limited field of view (FOV). The HRI essentially mimics an ultra-fast shutter, activated by applying a voltage pulse to a photocathode that is located in front of a microchannel plate. The shutter-open time (gate width) is determined by the length of the voltage pulse, which is on the order of $1 \mathrm{~ns}$, and the propagation of such short voltage pulses places constraints on the size of the detector, which typically have a diameter of $18 \mathrm{~mm}$. Combining this with the GOI spatial resolution of $\sim 20 \mathrm{lp} / \mathrm{mm}$, the effective number of pixels of the GOI is limited to $\sim 10^{5}$ (c.f. a typical CCD that has $\sim 10^{6}$ pixels). Using an appropriate magnification to preserve the overall spatial resolution of the microscope results in a FLIM FOV that is $\sim 10$ times smaller than that of a typical CCD camera. For the application considered here, this increases the chance that a randomly chosen FOV will be empty or contain very few cells, which is particularly problematic if the cells have been seeded at relatively low density. Imaging at lower magnification will increase the FOV size at the expense of spatial resolution but this is not desirable if sub-cellular resolution is required, e.g. for HCA. To try and ensure that each acquisition provides a useful image with cells in the FOV, we have developed an auto-find algorithm that acquires a single time gated image at the start of each acquisition and, if there are no cells in the FOV, the instrument moves to an adjacent FOV within the same well. To avoid skewing biological data, the algorithm does not look for the brightest cells, just for the presence of cells. For situations where it is desirable to have multiple cells in the field of view, e.g. for robust highthroughput small molecule screening, statistically significant results can be achieved by setting a user-chosen minimum number of cells in the FOV before it stops searching. In addition, it is possible to acquire multiple FOV per well to achieve requisite cell numbers.

Figure 2 shows data acquired from multiwell plate arrays of fixed cells stained with the green fluorescent nucleic acid stain, Syto 21. The conventional fluorescence intensity image (a) displays a typical CCD FOV from within a single well, which is larger than an equivalent FOV using the GOI with the same resolution (inset). The fluorescence lifetime images (b) show data that was automatically acquired from six different wells of a 96 well plate and illustrate the ability of the system to find cells in 
samples with low confluence. The samples exhibit similar fluorescence lifetimes, as expected since they are similar cells with the same history. The total acquisition time for the six wells (including the automatic setting optimisation and cell finding steps) was 57 seconds, which corresponds to less than 10 seconds per well.

Figure 2: Data from cells in a multiwell plate stained with Syto 21: (a) conventional fluorescence intensity image acquired at $x 40$ without the GOI in place and (b) automatically acquired lifetime images from six wells. The lifetime values are quoted as the mean \pm standard deviation over every pixel in each image.

To demonstrate the potential of the instrument to assay protein-protein interactions, it was applied to image the activation state of the small GTPase K-Ras in COS cells using FLIM-FRET. Ras proteins are associated with oncogenesis and imaging interactions between Ras proteins and their effectors is of interest for research and drug discovery. In a similar manner to earlier studies, e.g. [8, 9], we assayed the binding of the K-Ras protein labelled with mRFP to a co-expressed effector binding domain of Raf (Raf-RBD) labelled with EGFP, upon stimulation with EGF. For these experiments, live COS cells were cotransfected with EGFP-Raf RBD and K-Ras mRFP in a 96-well plate, stimulated with EGF and then fixed. As a control, we also fixed cells that were not stimulated.

Error! Reference source not found. shows FLIM images corresponding to data acquired from wells with (a) non-stimulated and (b) stimulated cells. The data acquisition was fully automated - i.e. the instrument located the cells, set the camera exposure time, acquired the FLIM data and then moved on to the next well. The average total acquisition time per well was $\sim 20$ seconds, which would corresponds to a total of 30 minutes for a 96 well plate. The FLIM images show that the stimulated cell exhibits a lower lifetime at the membrane than the non-stimulated control, which we attribute to FRET and which is consistent with our earlier experiments [7].

Figure 3: Data from cells in a multiwell plate co-transfected with EGFP-Raf RBD and $K$-Ras $m R F P$, which were stimulated with EGF and then fixed. An image from a control well (a) exhibits a lifetime of $2.58 \pm 2.70 n$ s at the cell membrane whereas the membrane of a stimulated cell $(b)$ has a lifetime of $2.20 \pm 1.50 \mathrm{~ns}$.

Finally, figure 4 shows data acquired from live HEK293 epithelial cells in 3 wells from a 96 well plate. To facilitate live cell imaging, a heating element was installed and an enclosure was constructed around the microscope to maintain the cells at 37 degrees. The time frame of this experiment did not require an elevated concentration of $\mathrm{CO}_{2}$ to be maintained. The HEK293 cells were stained with the membranepartitioning dye di-4-ANEPPDHQ, which exhibits a fluorescence lifetime that is sensitive to membrane order [3], and imaged after treating them for 10 minutes with varying concentrations of methyl- $\beta$-cyclodextrin $(\mathrm{MbCD})$ in phosphate buffered saline (PBS). After 10 minutes the cyclodextrin solutions were replaced by PBS to stop the cholesterol depletion process. The fluorescence lifetime of the di-4ANEPPDHQ was observed to decrease as a function of cyclodextrin concentration, as expected since this reduces order in the cell membrane by extracting cholesterol. This trend is clearly shown in figure $4(\mathrm{~d})$. The acquisition time was $\sim 9$ seconds per well including all automation steps. 
Figure 4: Fluorescence lifetime images of live cells in a 96-well plate stained with di4-ANEPPDHQ acquired 10 minutes post Treatment with (a) $0 \mathrm{mM}$, (b) $7 \mathrm{mM}$ and (c) $21 \mathrm{mM}$ cyclodextrin. (d) Chart showing mean fluorescence lifetime averaged over three wells.

In conclusion, we present what we believe is the first publication of a high speed, optically sectioning, automated FLIM multiwell plate reader applied to fixed and live cells, which could have significant potential to accelerate molecular cell biology research and for HCA for drug discovery. In particular, the ability to undertake higher throughput FLIM-FRET should provide new insights into protein-protein interactions and possible therapeutic interventions. As well as implementing wide-field timegating combined with a Nipkow disc confocal microscope in an automated multiwell plate reader, we have addressed several practical challenges in order to enable high throughput unsupervised optically sectioned FLIM. These include the need to automatically locate cells distributed sparsely within wells and to automatically optimise the CCD camera integration times on a well by well basis in order to obtain statistically consistent results between wells. We have shown the applicability of this new instrument to FLIM-FRET with a model FRET system, imaging the binding of $\mathrm{K}$-Ras to the Raf-RBD in COS cells, and we have demonstrated the capability for application to live cells. We envisage that this instrument can be further extended for HCA applications to incorporate spectrally-resolved and polarisation-resolved FLIM, which will be the subject of future work.

The authors gratefully acknowledge useful discussions with Jonathan Hares and Tony Dymoke-Bradshaw at Kentech Instruments Ltd. This work was supported by the Technology Strategy Board (with AstraZeneca, GSK R\&D Ltd, Kentech Instruments Ltd, Imperial College London and PerkinElmer Life and Analytical Sciences as project partners) as well as the UK Biotechnology and Biological Sciences Research Council (BBSRC BB/E003621/1), Cancer Research UK and the European Community (Framework VI Integrated Project "Integrated technologies for in vivo molecular imaging" contract number LSHG-CT-2003-503259). D. M. Grant, D. M. Owen and S. Kumar acknowledge studentships from the Chemical Biology Centre, Imperial College London supported by the UK Engineering and Physical Sciences Research Council (EPSRC). The mRFP plasmid was a generous gift from the lab of Professor R.Y. Tsien, University of California, San Diego. 
ND filter

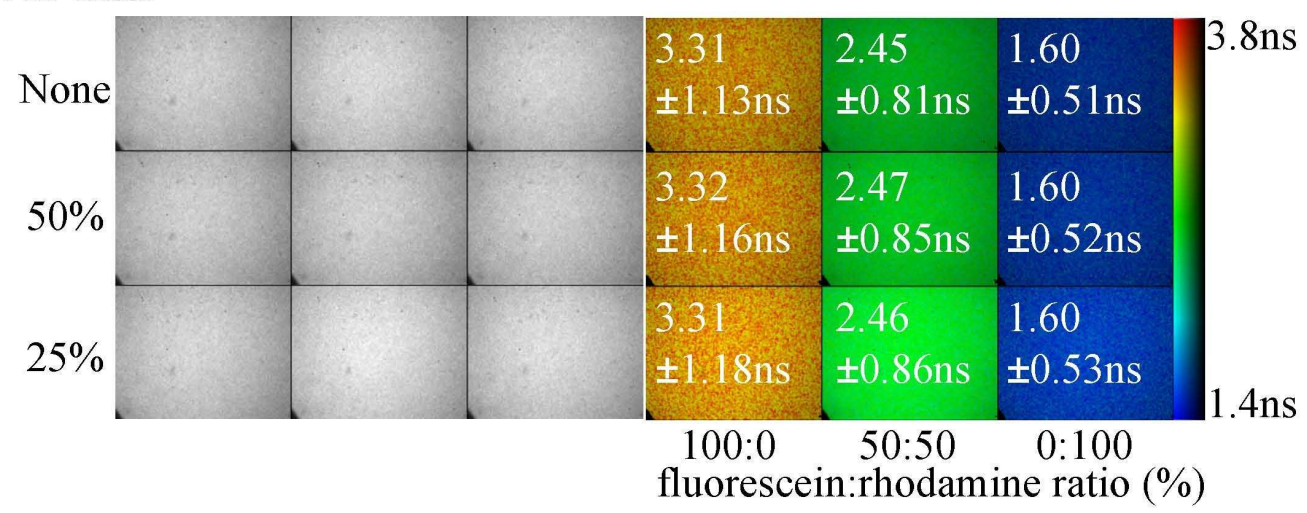

(a)

(b)

Figure 1: A montage of (a) time-gated and (b) FLIM images from nine separate acquisitions of fluorescein and rhodamine $B$. The fluorescein to rhodamine $B$ ratio changes from left to right as indicated and the fluorescence intensity was decreased by placing ND filters in the beam path. The first time gated images (a) have similar intensity and the lifetime maps (b) show consistent data in each column. 


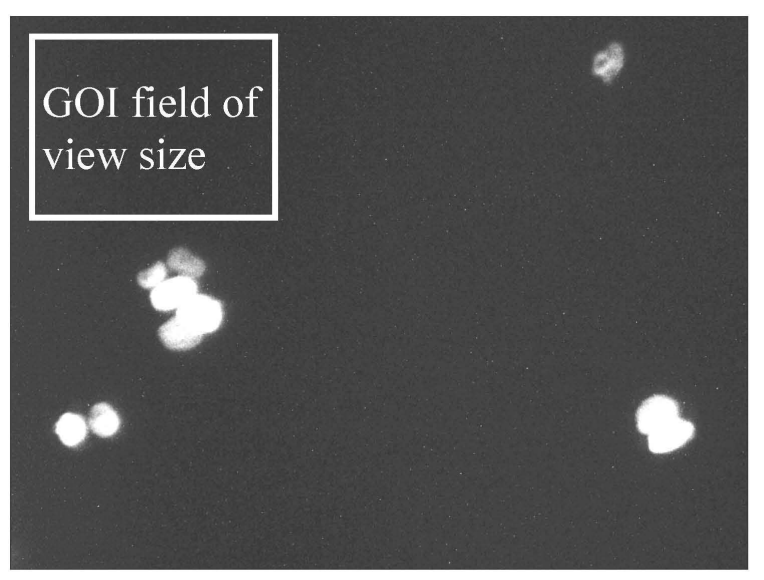

(a)

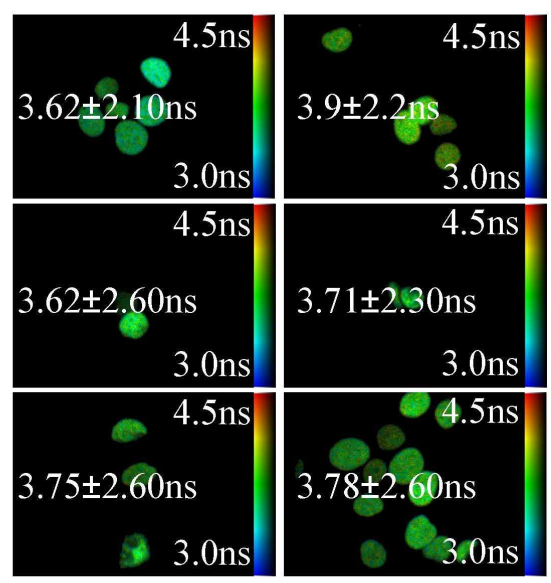

(b)

Figure 2: Data from cells in a multiwell plate stained with Syto 21: (a) conventional fluorescence intensity image acquired at $x 40$ without the GOI in place and (b) automatically acquired lifetime images from six wells. The lifetime values are quoted as the mean \pm standard deviation over every pixel in each image. $126 \times 62 \mathrm{~mm}(523 \times 523 \mathrm{DPI})$ 


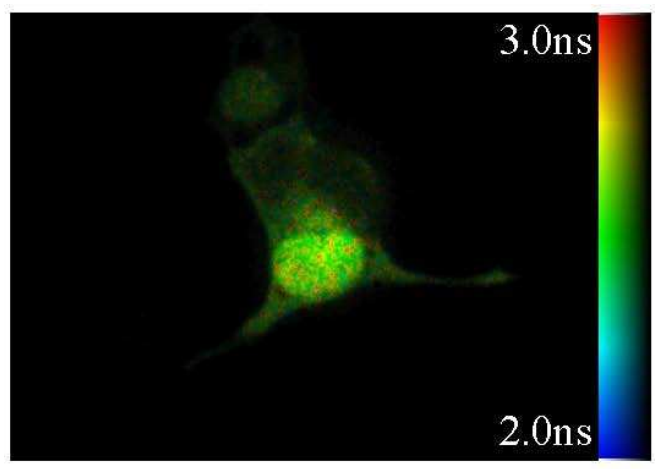

(a)

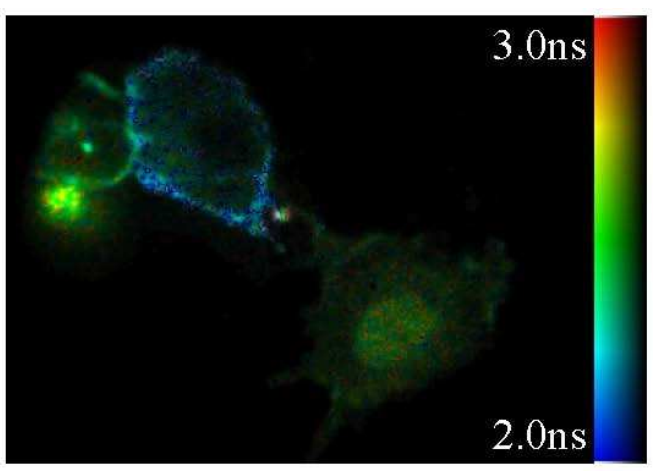

(b)

Figure 3: Data from cells in a multiwell plate co-transfected with EGFP-Raf RBD and K-Ras mRFP, which were stimulated with EGF and then fixed. An image from a control well (a) exhibits a lifetime of $2.58 \pm 2.70$ ns at the cell membrane whereas the membrane of a stimulated cell (b) has a lifetime of $2.20 \pm 1.50 \mathrm{~ns}$. $125 \times 52 \mathrm{~mm}(215 \times 215 \mathrm{DPI})$ 


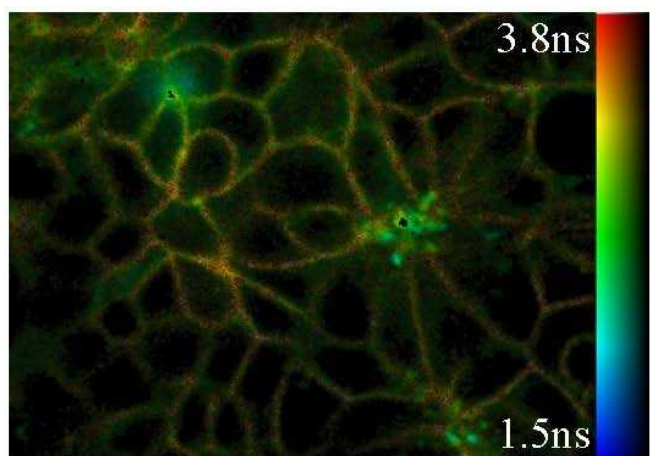

(a)

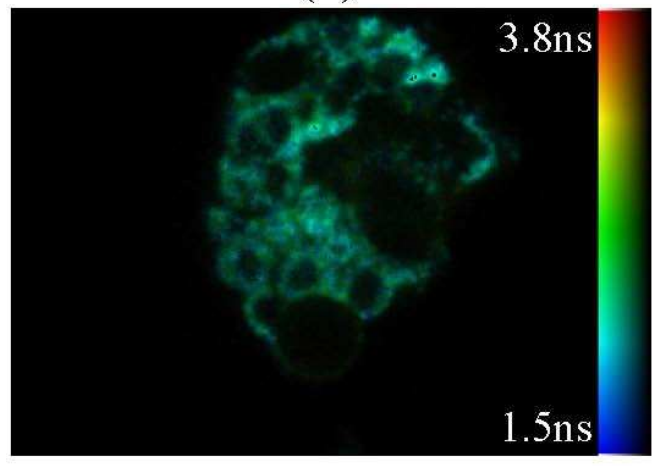

(c)

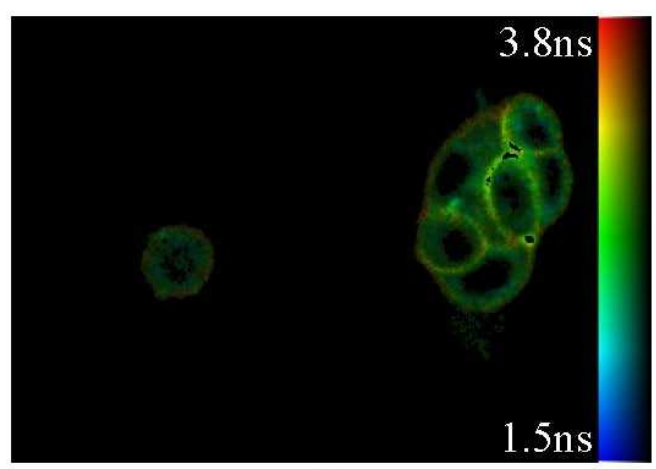

(b)

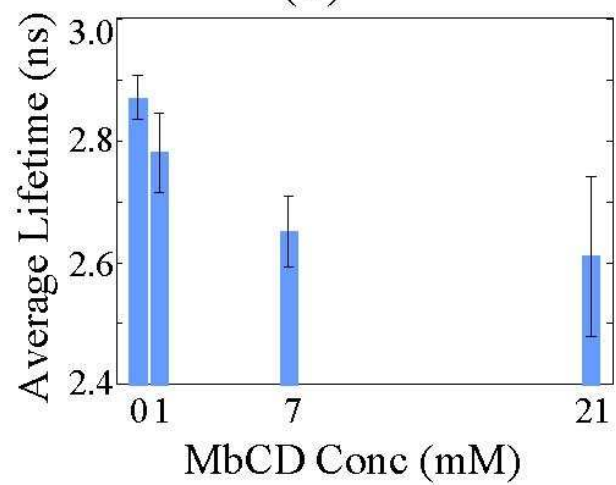

(d)

Figure 4: Fluorescence lifetime images of live cells in a 96-well plate stained with di-4-ANEPPDHQ acquired 10 minutes post Treatment with (a) $0 \mathrm{mM}$, (b) $7 \mathrm{mM}$ and (c) $21 \mathrm{mM}$ cyclodextrin. (d) Chart showing mean fluorescence lifetime averaged over three wells. $125 \times 104 \mathrm{~mm}(215 \times 215 \mathrm{DPI})$ 